\title{
REVISIÓN DE LAS CLASIFICACIONES DOCUMENTALES BASADAS EN EL SOPORTE
}

\author{
Blanca Rodríguez Bravo*
}

Resumen: Partiendo de la consideración del documento, desde un punto de vista estructural, como soporte portador de un mensaje, se abordan los diversos criterios sistematizadores que se han venido utilizando para tipificar el documento, para a continuación centrarse en dos de ellos: las tipologías documentales en función del soporte y en función del medio de transmisión del mensaje. Se concluye la necesidad de revisar estas clasificaciones debido a la introducción en el panorama documental del documento digital. La principal oposición no es ya la existente entre documentos librarios y no librarios, textuales o no textuales, sino la relativa a los documentos analógicos y digitales. Se estudian las principales diferencias entre ambos para terminar proponiendo nuevos criterios tipológicos y una clasificación.

Palabras clave: documentos, tipologías documentales, soportes documentales, documentos analógicos, documentos digitales, materiales no librarios, materiales especiales.

Abstract: Starting from the consideration of the document from a structural point of view, as support carrier of a message, we explain the diverse approaches to systematize the document, particularly considering the documentary classifications regarding the support carrier and the message transmission media. We conclude there is a need to revise these classifications due to the introduction of the digital document in the informative scene. The main opposition at present is not that between book and non-book materials, textual or non- textual documents, but that between analogic and digital documents. The differences between the latter are studied, and finally a new typology and classification are proposed.

Keywords: documents, classifications, documentary supports, analogic documents, digital documents, non book materials, special materials.

\section{Componentes documentales y criterios tipológicos}

El concepto de documento puede ser abordado desde distintos puntos de vista. El primero, mediante una aproximación funcional en la que se estudie su origen y evolución a lo largo del tiempo. El documento surge por la necesidad que el ser humano experimenta de registrar los hechos para superar así las limitaciones espacio-temporales. Al logro de este objetivo han contribuido la aparición de la imprenta primero y del documento digital después.

* Área de Biblioteconomía y Documentación. Facultad de Filosofía y Letras. Universidad de León. correo-e: dphbrb@unileon.es

Recibido: 17-1-2002. 
El segundo punto de vista sería el que correspondería a una aproximación estructural en la que se estudien los componentes del documento. Éstos son dos: el mensaje y el soporte que lo vehicula. Este soporte debe ser accesible y permitir la reutilización del contenido que guarda. En este sentido es una realidad ineludible que el nuevo documento digital tiene una menor estabilidad que los documentos anteriores, la permanencia del soporte, cualidad tradicional del documento «fuente permanente de información permanente» según Desantes (1), es cuestionada en el documento digital, y sobre todo en el que se transmite vía web.

Como dice Martín Vega (2) todo documento incluye tres tipos de componentes:

a) Componentes físicos o materiales. Todo documento se asienta en una determinada clase de soporte material. Ofrece, por consiguiente, cualidades de peso, tamaño, sustancia material, etc.

b) Componentes formales. Los documentos adquieren una estructura. La materia básica que los sustenta se dispone de una cierta manera (grabando sobre piedra, dando forma gráfica con la tinta, digitalizando datos en un ordenador...), con objeto de mostrar un contenido, de adquirir un sentido, de tener un significado, de transmitir un conocimiento.

c) Componentes conceptuales. Todos los documentos proporcionan un significado.

Los componentes físicos o materiales serían el soporte, los formales el medio de fijación del mensaje al soporte y, por último, los componentes conceptuales el contenido.

También según López Yepes (3), el documento tiene una triple dimensión, que sirve para fijar su tipología:

1) Soporte físico.

2) Mensaje informativo.

3) Posibilidad de transmisión o difusión del conocimiento sustentado en el documento y actualizado en el proceso documental.

La fijación del mensaje en un soporte permite, precisamente, que ese contenido pueda ser recuperado y reutilizado por el usuario, hecho que facilitan las unidades informativas al someter los documentos al proceso informativo-documental.

Los criterios que pueden utilizarse para sistematizar el documento, en función de sus componentes, son diversos; sirvan para ello de ejemplo los criterios que López Yepes ha establecido (4):

a) Por la forma de representación del mensaje en el soporte físico:

- Gráfico: libro, revista, etc.

- Iconográfico: fotografía, pintura, etc.

- Fónico: disco, cinta magnetofónica, etc.

- Audiovisual: película, vídeo, etc.

- Plástico: objetos.

- Electrónico: disquete, disco óptico digital, etc. 
b) Por el nivel de difusión:

- Publicado: cualquier documento multiplicado en número suficiente de ejemplares que permiten su difusión pública.

- Inédito: manuscrito o documento de archivo no publicado.

- Reservado: documento manuscrito o impreso pero no difundido.

c) Por el grado de originalidad en su creación:

- Fuentes: los documentos más cercanos a las informaciones o acontecimientos que reflejan o que constituyen la materia prima - documentos de época, crónicas, estadísticas, legislación, objetos de museo, etc.

- Bibliografía: los documentos elaborados desde las fuentes - monografía, artículo de revista, etc.

d) Por el grado de modificación de la naturaleza del mensaje, resultado del análisis documental:

- Primario: libro, artículo de revista, etc.

— Secundario: ficha bibliográfica, repertorio bibliográfico, resumen, etc.

e) Por el grado de transformación del mensaje documentario soportado en el documento:

- Mensaje documentado.

- Mensaje marginal.

- Mensaje referencial.

- Mensaje documental.

f) Por su situación en el sistema de las ciencias:

- Jurídico, matemático, médico, etc.

g) Por el grado de comprobación de la verdad del mensaje:

- Científico: monografía científica, tesis doctoral, etc.

- No científico: artículo de prensa, ensayo, etc.

López Yepes evita establecer una sistematización en función del soporte, seguramente por la poca estabilidad que estas clasificaciones tienen. Como dice Desantes (5): «la teoría de los soportes en la documentación va a constituir siempre una síntesis incompleta, susceptible permanentemente de ampliación en todas las direcciones».

Sí distingue Martínez Comeche (6) entre el criterio del soporte y del medio empleado para fijar el mensaje a ese soporte. Establece diversas clasificaciones, la mayoría coincidentes con las de López Yepes, que inciden en:

1) La naturaleza del soporte.

2) El código empleado en el mensaje.

3) El rigor científico del mensaje.

4) El área del conocimiento que abarca el mensaje.

5) El tratamiento y consiguiente modificación del mensaje original.

6) La capacidad de difusión. 
1) Desde la perspectiva del soporte empleado, los documentos utilizan hoy día mayoritariamente el papel (libros, artículos, folletos...), los materiales magnéticos (cintas magnetofónicas y de vídeo, disquetes...) y los soportes ópticos (videodiscos, CD-ROM, discos compactos...), aunque a lo largo de la historia han variado mucho (madera, piel, pergamino, piedra, metal...).

2) La clasificación atendiendo al código empleado en el mensaje distingue entre documentos textuales (cuando los signos corresponden a la lengua escrita), gráficos (mapas, planos), iconográficos (cuadros, diapositivas, fotografías), sonoros o fónicos (cintas, discos), audiovisuales (películas, vídeos), plásticos o tridimensionales (cualquier objeto conservado en un museo, por ejemplo), informáticos (legibles por ordenador), o documentos multi-media (cuando el documento combina varios de los códigos anteriormente expuestos).

3) En cuanto al rigor científico del mensaje, suelen dividirse los documentos en científicos, técnicos y de divulgación, según va disminuyendo el nivel de profundidad y precisión del mensaje emitido.

4) El documento puede ser también agrupado de acuerdo con el área de conocimiento que abarca en el sistema de las ciencias, hablándose entonces de documentos económicos, sociales, históricos, jurídicos o lingüísticos, entre una enorme variedad de mensajes posibles.

5) La estructuración del documento, que considera el tratamiento a que es sometido el contenido del mensaje, modificando consiguientemente su presentación, diferencia entre un documento primario, un documento secundario y un documento terciario. No existe acuerdo entre los estudiosos sobre el documento terciario.

6) Por último, en lo que respecta a su capacidad de difusión, el documento puede ser portador de un mensaje publicado o público, inédito, reservado o personal.

\section{Las tipologías documentales según el soporte: los documentos librarios y no librarios.}

Como dicen Fothergill y Butchart (7) es comúnmente aceptado que la función de una biblioteca es la colección, conservación, organización y uso de los documentos, y han sido los documentos impresos los que han monopolizado las bibliotecas hasta nuestros días. Ciertamente, los documentos bibliográficos son todavía mayoritarios pero su protagonismo disminuye. Hoy día, como señala Ruiz de Osma (8), los recursos de información de que disponemos no son solamente libros, publicaciones periódicas, publicaciones de congresos, etc., existen también otros documentos como mapas, gráficos, diapositivas, vídeos, películas, soportes ópticos, etc. y que «desafortunadamente no tienen un nombre concreto para designarlos. Estos productos reciben distintas denominaciones: soporte no librario, material especial, material no librario, material me- 
nor, etc. Cuando se habla de estos tipos de documentos siempre aparece una cierta inseguridad relativa a la terminología empleada». Como dicen Fothergill y Butchart (7) «se trata de una materia sobrecargada de jergas y tecnicismos donde abundan tanto los términos específicos como los generales». Ponen de relieve que la incertidumbre y la confusión aparecen siempre que se habla sobre terminología relativa a materiales no librarios. Su implicación con el mundo de la televisión, los ordenadores y la edición hace que el panorama se complique aún más.

No existe ninguna normativa al respecto $\mathrm{y}$, por lo tanto, no hay acuerdo general en emplear una terminología determinada. Así pues, los autores anglosajones utilizan denominaciones como non-book material, non-book media, non-print material, etc. para designarlos, estableciendo una clara distinción entre los materiales impresos y no impresos y los que tienen forma de libro y los que no. Como dice Meyriat (9) los bibliotecarios anglosajones han inventado el término «non-books» para designar todos los objetos que se confiaban a sus cuidados pero que no sabían como tratar. Esta misma agrupación la realizó la norma de descripción bibliográfica ISBD (NBM) de 1977 (10). Los inconvenientes de esta práctica se dejan ver rápidamente como los de todas las que reposan sobre una definición negativa, por exclusión. Los productos así agrupados no tienen en común mas que el estar recubiertos por la misma etiqueta; y el falso conjunto que constituyen es reducido progresivamente a medida que alguno de sus elementos gana en importancia suficiente como para requerir un tratamiento propio.

Para establecer un consenso se deben tomar como punto de partida los trabajos publicados sobre la materia. ¿Qué términos aparecen en la literatura general sobre soportes no librarios? Si se pasa revista a los títulos de los libros sobre el tema se llega a la conclusión de que no parece existir un acuerdo general en uno solo de los términos usados. No existe tampoco acuerdo sobre los conceptos subyacentes en ellos, como vamos a ver a continuación.

Uno de los términos más utilizados en castellano para designar a estos productos es material especial, pues ha sido el elegido por las Reglas de Catalogación Españolas (11) para agrupar a los documentos que no eran monografías ni publicaciones seriadas, pero, como bien argumenta Carrión (12), «el adjetivo especial adjudicado a aquellos materiales no bibliográficos es cada vez menos intenso porque la biblioteca se abre de continuo a nuevos medios y el tratamiento catalográfico de todos ellos es cada vez más homogéneo». De cualquier modo, el término más empleado es material no librario, traducción poco precisa de los términos anglosajones y que, como éstos, sigue siendo una denominación vaga y confusa. A este respecto señala el mismo autor que «en la expresión non book material se incluyen tanto los materiales impresos como los no impresos, los materiales que necesitan ayuda mecánica para acceder a su contenido y los que no necesitan esta ayuda».

Sin embargo, los documentos que más se identifican con este concepto de «no librarios» son los audiovisuales; así lo confirman Carrión y López de Quintana. Según Carrión (12), «son éstos los materiales que propiamente pueden llamarse no librarios, ya que publicaciones seriadas, manuscritos, mapas, música impresa, grabados pueden adoptar, y de hecho adoptan, muchas veces la forma de libro». López de Quintana (13) opina igualmente que si bien en el mundo anglosajón se denomina non-books a todo el conjunto de documentos que no están impresos, el material por excelencia asimilado a este concepto es el soporte vídeo, o, más exactamente, la información audiovi- 
sual y el concepto de documento audiovisual se aplica de forma genérica tanto a las cintas de vídeo o audio por separado, como a los títulos formados por soportes mixtos que mezclan información escrita con los soportes alternativos: vídeo, registros sonoros o diapositivas.

Resulta difícil ofrecer una definición adecuada para este tipo de documentos, Amalia Sarriá (14) considera material no librario «todos los materiales que se apartan de la forma tradicional de libro o revista, incluyendo en este concepto tanto los documentos especiales bibliográficos, cuyo soporte es el papel, como los documentos especiales no bibliográficos que transmiten la información en forma de imágenes y sonidos en un soporte distinto de las clásicas materias escriptóreas».

También el Glosario ALA (15), cuando se refiere a non-book material remite a «materiales audiovisuales», y los define como «aquéllos que transmiten información mediante el sonido y la imagen en lugar de hacerlo utilizando texto escrito. Gran parte de este material requiere el empleo de un equipo especial para oírse o verse y recibe la denominación de material no impreso porque no se hace para ser leído».

Fotherhill y Butchart (7) proponen como definición de material no librario: «los que no forman parte de un libro» y excluyen cualquier mensaje impreso que se presente en forma de manuscrito, mapa, publicación periódica, folleto o partitura. Todos los documentos impresos serían documentos librarios aunque no tengan el formato del libro, como es el caso de los mapas. Añaden a continuación que el concepto de materiales no librarios «abarca un amplio espectro puesto que también incluye los materiales excluídos anteriormente, siempre que se presenten de forma diferente, como un mapa en una diapositiva o una publicación periódica en una microficha».

Buonocore (16) distingue entre «materiales audiovisuales o fonovisuales» $\mathrm{y}$ «materiales especiales». Los primeros serían los medios visuales (diapositivas, etc.), auditivos (discos, cinta magnética) y audiovisuales (cine, televisión), según impresionen al sentido de la vista, el del oído o ambos a la vez. Estos documentos necesitan aparatos mediadores. Los «materiales especiales» serían los documentos que no son libros propiamente dichos pero que no necesitan el concurso de ningún aparato, siendo de utilización directa e inmediata: folletos, periódicos, música impresa, mapas, planos, láminas, etc.

Ruiz de Osma (8) considera materiales no librarios aquellos documentos especiales bibliográficos presentados en soporte papel y los no bibliográficos cualquiera que sea el soporte en que se presenten, y propone la siguiente clasificación:

1. Soporte papel: fotografías, partituras musicales, dibujos, grabados, carteles, material cartográfico.

2. Película: microformas, diapositivas, películas animadas.

3. Material plástico: transparencias, discos de vinilo.

4. Soporte magnético: casetes, vídeos, discos magnéticos.

5. Sistemas de almacenamiento óptico: CD-A, CD-ROM, CD-I, DAT, CD ROM $\mathrm{XA}, \mathrm{CD}-\mathrm{V}, \mathrm{CD}+\mathrm{G}, \mathrm{CD}-\mathrm{ASSIST}$, etc.

Fothergill y Butchart (7) coinciden con Ruiz de Osma en los cuatro materiales de que los documentos hacen uso:

a) Papel, con el que pueden hacerse tarjetas, reproducciones de arte, fotografías impresas. 
b) Película, que incluye: tira de filmina, diapositiva, cinefilm (35 mm, $16 \mathrm{~mm}$ ), microforma (rollo de $35 \mathrm{~mm}$, de $16 \mathrm{~mm}$, tarjetas de apertura, microficha).

c) Cinta magnética, que incluye: cinta sonora (bobina, casete), cinta de vídeo (bobina, casete), discos magnéticos.

d) Plástico, bien plano y transparente, u opaco y con surcos: plástico transparente (transparencias utilizadas en un retroproyector), discos de vinilo (discos de gramófono), sistemas de almacenamiento óptico (disco compacto musical, CDROM).

Los mismos autores dejan constancia de que en proyectos piloto se utiliza también otro tipo de materiales: maquetas, modelos, o animales disecados, que también se consideran materiales no librarios. Existen también mezclas de materiales, conjuntos de multimedios, que se llaman «kits». A estas clasificaciones se añadiría hoy el documento web.

Como se puede deducir de lo anteriormente expuesto, no sólo no existe unanimidad en los adjetivos que pretenden englobar a los documentos cuyo formato se diferencia del libro: no librarios, no bibliográficos, no impresos, especiales o audiovisuales, sino que tampoco existe en los sustantivos que los acompañan: los términos materiales y medios se imponen a los de documentos o soportes, mucho más adecuados, sin embargo. Entendemos que el término material puede tomarse como equivalente al de soporte pues se refiere a éste; medio alude al sistema por el que el mensaje se fija al soporte y sobre este componente del documento trataremos a continuación. Ambos términos son restrictivos pues prescinden del principal componente del documento: el mensaje. Preferimos la utilización del término soporte para hacer referencia al mero vehículo portador del mensaje y el término documento como integrador de continente más contenido.

En conclusión, cuando se habla de documentos, o más frecuentemente materiales, no librarios, generalmente se hace referencia a los documentos sonoros, visuales y audiovisuales, que son los que más se alejan del libro, tanto por su formato como por el medio de fijación del mensaje al soporte. No obstante, a veces se integran también todo tipo de documentos impresos, en soporte papel, bibliográficos y gráficos, distintos al libro y a la publicación seriada. Este último sentido amplio es el que suele darse también a la expresión materiales especiales, y de ello son reflejo nuestras normas de catalogación (11).

Existe, por tanto, una gran confusión que tiene dos orígenes: por un lado, el afán de agrupar bajo una única denominación documentos que poco tienen en común, y, por otro, el hecho de que se mezclan dos criterios tipificadores: el del soporte o material que sirve para vehicular el mensaje y el del medio o código por el que el mensaje se fija a ese soporte.

\section{Las tipologías documentales en función del medio de transmisión del mensaje: los documentos textuales y no textuales}

Son muchos y variados los soportes documentales, y además de la distinción entre documentos librarios y no librarios, «normales» y «especiales», otras agrupaciones que se suelen hacer no hacen ya referencia al soporte sino al medio y código que se 
utiliza para transmitir el mensaje. Entendemos que la sistematización en función del código empleado tiene mayor trascendencia en Documentación, pues los distintos códigos y las diferentes disposiciones de los mensajes en los soportes condicionan el tratamiento de los contenidos y su recuperación. Los códigos son los siguientes:

- En el caso de que sea la escritura, nos encontramos con los documentos textuales o escritos, hasta ahora en papel fundamentalmente, con lo que equivalían a los bibliográficos o impresos, pero en la actualidad lo textual tiene también mucha presencia en los documentos digitales.

- Si es el sonido, nos encontramos con los documentos sonoros que engloban discos, cassettes y CD-Audio, principalmente.

- Si se trata de la imagen, fija o en movimiento, se agrupan las fotografías, películas, diapositivas, etc.

- Existen por último los documentos mixtos, hasta hace poco audiovisuales exclusivamente, pero el documento digital permite la integración del documento sonoro, visual y textual, y aunque es este último el que predomina, cada vez la tendencia al multimedia es mayor.

Martín Vega se fija también en el medio de transmisión del mensaje para tipificar el documento y dice que formalmente el documento puede ser (2):

1. Textual. Presenta información de manera escrita: libros, publicaciones periódicas, documentos administrativos, bibliografías, etc. Necesita un conocimiento del sistema ordinario de lectura, y se lee directamente, sin necesidad de ninguna herramienta intermedia entre el documento y nuestra vista.

2. Especial. Puede incluir una parte de texto pero su información esencial aparece con otro tipo de signos. Además, su estructura es muy variable ya que depende de la naturaleza del soporte: plástico, arcilla... Debe verse, oírse o manipularse. Si solamente se lee, necesita un tipo de lectura especial no alfabética, por ejemplo, un libro de partituras musicales, o, en otros casos, precisa de un lector que sirve de intermediario. Entre los documentos de carácter especial se incluyen:

- Los de tipo iconográfico. Documentos librarios o no librarios que representan imágenes gráficas: fotografías, cuadros, mapas, planos, gráficos, etc.

- Plásticos. Presentan objetos en general moldeables: esculturas, maquetas, monumentos, etc.

- Sonoros. Son documentos cuya información ordinariamente se transmite a través de instrumentos sonoros. Constituyen formas destinadas fundamentalmente a ser interpretadas o descifradas por el oído: discos, cintas grabadas, etc.

- Mixtos. Reúnen las mismas informaciones bajo diversos tipos de formas en un solo documento o en varios, por ejemplo, libro-disco, libro-casete, conjuntos pedagógicos, etc.

- Audiovisuales. Son documentos mixtos, caracterizados por la combinación de sonido e imagen: películas, videodiscos.

- Informáticos. Utilizan sistemas electrónicos para la realización de lecturas me- 
diante procedimientos óptico-magnéticos. A menudo constituyen programas que permiten realizar diversos trabajos como escritura, cálculo, selección, manejo de fondos bibliográficos, etc. Estos soportes, conocidos también como kits multimedia, conservan y transmiten al menos tres clases de signos: textuales, sonoros y gráficos.

Para Martín Vega, por tanto, serían documentos especiales aquéllos cuyo código no es exclusivamente el lenguaje escrito; es, por tanto, un concepto de gran amplitud semántica, como ya habíamos manifestado.

Antes de terminar este apartado, procede reflexionar sobre la frecuente utilización del sustantivo material en vez de documento, pues ello parece indicar que estos tipos documentales han tenido una categoría inferior en las bibliotecas. En este sentido, Fothergill y Butchart (7) opinan que existe la creencia de que en algún sentido los libros tienen valores intelectuales y espirituales superiores. "Se considera normal sentarse al sol un día de verano y leer un libro, mientras que sentarse a ver un programa de televisión en un televisor portátil en la misma pradera y bajo el mismo sol, se interpreta como un síntoma de holgazanería».

La UNESCO (17) ya en 1968 determinaba, por ejemplo, que las películas tienen la misma categoría intelectual que los libros, los periódicos y las revistas, y deben beneficiarse de todas las medidas que se tomen para el desarrollo de la cultura, el progreso tecnológico y el crecimiento intelectual y moral de la humanidad, sin discriminarse en ningún caso por la forma material en que las obras se producen o se transmiten.

No hay duda de la superioridad del documento escrito como vehículo del pensamiento, pues es el código verbal el que los hombres utilizamos fundamentalmente, pero el documento sonoro o el documento de imagen -fija o de movimiento-, y el documento audiovisual, se presentan como más idóneos para reproducir otros mensajes o completar el escrito. Como dicen Fothergill y Butchart (7) «el canto de un jilguero se puede describir con palabras, pero una grabación lo describe mucho mejor. Las fotografías del feto ayudan a entender el milagro del nacimiento de una forma que las palabras simplemente no pueden igualar».

Uno de los inconvenientes de los documentos no librarios es la necesidad de mediación de artefactos para acceder al contenido, y la gran cantidad de aparatos y equipos que han aparecido. Para solucionarlo, hay un constante esfuerzo de reducción de los equipos a formatos que valgan para todas las funciones sin perder la versatilidad que da la variedad. Con la reducción del espacio, también aparecen aparatos más manejables y portátiles, al menos en algunos casos. La tendencia actual es a unificar formatos que permitan la conservación de los contenidos documentales, y a buscar sistemas cada vez más ergonómicos que permitan a los distintos tipos documentales competir con el todavía rey del mercado de la edición: el libro.

El documento digital, con su inmensa capacidad de almacenamiento, que supera a la de las microformas, y cuyo soporte transformable permite el acceso a distancia a sus contenidos y una fácil manipulación es, en los comienzos del tercer milenio, una realidad con un peso específico que entra a competir con los soportes tradicionales, entre los que incluimos tanto los bibliográficos como los no bibliográficos. 


\section{Documentos analógicos versus documentos digitales}

Las diversas clasificaciones tipológicas que se han establecido utilizando como criterio el soporte deben ser revisadas, pues la introducción del documento digital ha hecho que el panorama dé un vuelco considerable.

Hasta hace una década, la principal oposición se establecía entre los documentos en papel, que dominaban el mercado de la edición, a los que se denominaba documentos impresos, bibliográficos o librarios normalmente, y el resto de los documentos, que eran producto de los avances técnicos producidos en el siglo $\mathrm{xx}$, y que se agrupaban, a efectos prácticos, bajo denominaciones en absoluto acertadas: documentos especiales, no impresos o no librarios, traduciendo de las expresiones inglesas «nonprint materials»o «non-book materials», como hemos visto.

Según Codina (18), la oposición más significativa no es ya, sin embargo, la que existe entre medios electrónicos (los audiovisuales) y no electrónicos (los bibliográficos) sino la que hay entre medios analógicos y medios digitales, entre átomos y bits en terminología de Negroponte (19). No obstante, conviene señalar que los adjetivos «electrónico», «digital» e «informático» también se intercambian. El concepto de documento electrónico es más amplio y englobaría al documento digital o informático y al audiovisual.

Lo que caracteriza a los medios analógicos es que representan la información mediante una relación de parecido o de analogía: se mantiene cierta semejanza entre la información y su codificación, aunque sea remota. En cambio, en los medios digitales, que utilizan series de bits para representar la información, cualquier semejanza entre la información y su representación queda destruida. Lo mismo un texto que una fotografía o un vídeo, son series de ceros y unos para el ordenador, es decir que se representan de la misma manera.

En los medios analógicos, sin embargo, cada morfología o código y cada soporte de la información exige una forma de codificación propia. De hecho, ningún soporte analógico resulta adecuado para todas las morfologías de la información al mismo tiempo. Por el contrario, los medios digitales pueden contener cualquier morfología de la información y cualquier combinación entre ellas (18).

El documento digital tiene también otras características peculiares, puestas de relieve por Linda Schamber (20): es fácilmente manipulable, es enlazable interna y externamente, es rápidamente transformable, es fácilmente accesible, instantáneamente transportable e infinitamente replicable.

De estas características se deduce que la primera diferencia de los documentos digitales con respecto a los analógicos estriba en que en ellos se produce una disociación entre el soporte y el contenido. Las peculiaridades establecidas por Schamber se refieren a los contenidos, los soportes han perdido relevancia, de hecho, los mensajes que contienen se copian con facilidad en otro soporte, lo que los convierte en transformables o manipulables y en transportables.

La separación entre contenido y soporte hace que estos documentos sean compuestos (distintos códigos), distribuidos (en varios ficheros) y dinámicos (fácilmente modificables). De estas características se derivan las nuevas posibilidades que ofrecen, que son muchas. Sus puntos fuertes son su gran capacidad de almacenamiento, su virtualidad y accesibilidad a distancia y su actualización. Los débiles son la dificultad de conservación de sus mensajes (su duración) y la mayor dificultad que presentan para garantizar su integridad y autenticidad. La facilidad que tienen los mensajes para cam- 
biar de soporte y su sencilla manipulación están en el origen tanto de sus ventajas como de sus inconvenientes.

Volviendo a la clasificación según el soporte, Sagredo (21) distingue en función de su materialidad y de la intermediación para acceder a su contenido, tres tipos de documentos que denomina, real, irreal y virtual:

- El documento real sería aquél con un soporte material sobre el que se inscribe o reposa un documento decodificable directamente por los sentidos. El documento bibliográfico, que surge con la aparición de la escritura, sería el documento real por excelencia.

- El documento irreal cuenta también con un soporte material pero el usuario no puede acceder directamente a la información contenida, necesita la intermediación de aparatos lectores. Se trata de un documento ya utilizado desde finales del siglo XIX: el microfilm, las diapositivas, los discos, casetes, vídeos y también los disquetes, el CD-ROM y el DVD.

- Por último, Sagredo entiende por documento virtual aquél del que no disponemos in situ, ni del soporte tangible, ni del mensaje, y que para acceder a este último necesita de los periféricos y de recursos adecuados que permitan entrar en cualquier momento en el depósito irreal, donde se conservan todos los documentos.

Estos documentos virtuales, accesibles y manipulables a distancia por procedimientos on line y en tiempo real, son también fácilmente copiables, con lo que con el mismo contenido obtendríamos un documento tangible —irreal—, o tangible y decodificable directamente -real-.

Como dice Sagredo (21) el documento virtual está en potencia en todos y cada uno de los depósitos a los que accedemos en Internet, con la peculiaridad de que a partir de él el usuario puede crear un nuevo ejemplar documental, que a su vez puede ser almacenado, y así sucesivamente. El usuario se puede convertir fácilmente en creador y emisor en el proceso informativo documental, lo que es una ventaja indudable, pero que tiene como contrapartida la falta de permanencia del documento hasta ahora garantía referencial última.

El documento digital, en el que también todavía predomina lo textual, aún no ha conseguido superar al documento en papel en algunos aspectos, fundamentalmente en lo que a ergonomía se refiere, la comodidad y libertad que el acceso directo proporciona al documento en papel. No parece que vaya, por tanto, a suplantar en un plazo breve al documento analógico en general y bibliográfico en particular que es el que todavía ocupa una mayor cota del mercado de la edición. El libro sigue siendo hoy por hoy más fácil de manejar.

Esta característica, que creemos es fundamental para la supervivencia del documento bibliográfico, nos lleva a proponer, apoyándonos en Sagredo y Codina, unos criterios sistematizadores y una clasificación.

Los criterios son cuatro y de su combinación obtendríamos cuatro clases:

- Que exista analogía entre la información y su codificación, lo que divide a los documentos en analógicos y digitales.

- Que su soporte permita descifrar el mensaje directamente por los sentidos, o 
necesite la mediación de aparatos. Este criterio diferencia al documento bibliográfico de los demás.

- Que su soporte sea tangible o intangible. En este caso es el documento digital que se transmite por Internet el que se distingue de los restantes.

- Que su soporte sea estable o inestable. La estabilidad del soporte ha disminuido con la evolución de los tipos documentales.

Los grupos serían los siguientes:

1. Documentos analógicos directamente decodificables: serían los documentos gráficos y bibliográficos que no necesitan aparatos mediadores para acceder a su mensaje. Su soporte es el papel o similar, soporte tangible y muy estable.

2. Documentos analógicos que no son decodificables por los sentidos directamente sino que necesitan aparatos de lectura: diapositivas, vídeos, discos, etc. $\mathrm{Su}$ soporte es tangible y medianamente estable.

3. Documentos digitales cuyo soporte es tangible pero no de impacto directo, que necesita de un ordenador y lectores. Son el CD-ROM y el DVD. La estabilidad de su soporte es también mediana.

4. Documentos digitales cuyo soporte es intangible, el documento virtual, que no se decodifica directamente, también necesitado de ordenador y conexión a la red en este caso. Son los documentos que circulan por Internet, y son muy inestables.

Constatamos que estos cuatro tipos son también estadios en la evolución documental. En ella se observa la evolución tecnológica que el documento ha vivido y vive, pues no parece que el documento digital vaya a desplazar en un plazo corto a los anteriores y, curiosamente, parece que es el documento librario el que puede lograr mayores expectativas de vida: no fue desplazado por el audiovisual y todavía tampoco por el digital. Su ergonomía superior juega a su favor, junto con el hecho de que el texto es el principal vehículo del pensamiento.

\section{Bibliografía}

1. DESANTES GUANTER, J. M. ${ }^{\text {a }}$ Teoría y régimen jurídico de la documentación. Madrid: EUDEMA, 1987.

2. MARTÍN VEGA, A. Fuentes de información general. Gijón: TREA. 1995.

3. LÓPEZ YEPES, J. Teoría de la documentación. Pamplona: EUNSA, 1978.

4. LÓPEZ YEPES, J. La aventura de la investigación científica. Madrid: Síntesis, 1995.

5. DESANTES GUANTER, J. M. El valor jurídico de los novísimos soportes documentales. Revista General de Información y Documentación, 1992, vol. 2,n. ${ }^{\circ}$ 1, p. 17-31.

6. MARTÍNEZ COMECHE, J. A. Teoría de la información documental y de las instituciones documentales. Madrid: Síntesis, 1995.

7. FOTHERGILL, R. y BUTCHART, I. Materiales no librarios en las bibliotecas: guía práctica. (3. ${ }^{a}$ ed. orig.) Madrid: Fundación Germán Sánchez Ruipérez, 1992.

8. RUIZ DE OSMA DELATAS, E. Fuentes de información sobre materiales no librarios. En: TORRES RAMÍREZ, I. (coord.). Las fuentes de información: estudios teórico-prácticos. Madrid: Síntesis, 1998, p. 387-399.

9. MEYRIAT, J. Document, documentation, documentologie. Revue de Bibliologie, Schema et Schematisation, 1981, vol. 19, p. 25-36. 
10. IFLA. ISBD(NBM): International standard bibliographic description for non-book materials. London: British Library, 1977.

11. REGLAS DE CATALOGACIÓN. II, Materiales especiales. Madrid: Dirección General del Libro y Bibliotecas, 1988.

12. CARRIÓN GÚTIEZ, M. Manual de bibliotecas. Madrid: Fundación Germán Sánchez Ruipérez, 1990.

13. LÓPEZ DE QUINTANA SÁENZ, E. Fuentes para el material no librario. En: CARRIZO SAINERO, G. (editora.). Manual de Fuentes de Información., 2. ${ }^{a}$ ed. Zaragoza: CEGAL, 2000, p. 369-391.

14. SARRIÁ, A. Control de documentos especiales. Boletín de Documentación FIES, 1977, vol. 1, p. 113-129.

15. YOUNG, H. (ed). Glosario ALA de Bibliotecología y Ciencias de la Información. Madrid: Díaz de Santos, 1988.

16. BUONOCORE, D. Diccionario de bibliotecología. Buenos Aires: Marymar, 1976.

17. UNESCO. Text of the resolution adopted unanimously by the fifteenth session of the General Conference of UNESCO at its forty-second plenary meeting on Wednesday, 20th November 1968, at UNESCO House, París. International Conference on the Cataloging of Audiovisual Materials. London: IFTC, 1975.

18. CODINA, L. El libro digital y la WWW. Madrid: Tauro, 2000.

19. NEGROPONTE, N. El mundo digital. Barcelona: Ediciones B., 1995.

20. SCHAMBER, L. (1996). What is a document? Rethinking the concept in uneasy times. Journal of the American Society for Information Science, 1996, vol. 47, n. 9, p. 669-671.

21. SAGREDO FERNÁNDEZ, F. Documento y sistema virtual. En TRAMULLAS, J. (ed.), Tendencias de investigación en Documentación, Zaragoza: 1996, p. 9-17. 